\title{
A THEOREM ON A MAPPING FROM A SPHERE TO THE CIRCLE AND THE SIMULTANEOUS DIAGO- NALIZATION OF TWO HERMITIAN MATRICES
}

\author{
YIK-HOI AU-YEUNG ${ }^{1}$
}

1. Introduction and statement of the theorems. We denote by $F$ the field $R$ of real numbers, the field $C$ of complex numbers, or the field $H$ of real quaternions, and by $F^{n}$ an $n$-dimensional left vector space over $F$. If $A$ is a matrix with elements in $F$, we denote by $A^{*}$ its conjugate transpose. In all three cases of $F$, an $n \times n$ matrix $A$ is said to be hermitian if $A=A^{*}$ and unitary if $A A^{*}=I$, where $I$ is the $n \times n$ identity matrix. An $n \times n$ hermitian matrix $A$ is said to be positive definite if $u A u^{*}>0$ for all $u(\neq 0)$ in $F^{n}$. Here and in what follows we regard $u$ as a $1 \times n$ matrix and identify a $1 \times 1$ matrix with its single element.

The purpose of this note is to prove Theorem 1 on a mapping from a sphere to the circle, and use it to prove Theorem 2 on the simultaneous diagonalization of two hermitian matrices.

TheOREM 1. Let $A$ and $B$ be two $n \times n$ hermitian matrices with elements in $F$ such that $\left(u A u^{*}\right)^{2}+\left(u B u^{*}\right)^{2}>0$ for all $u(\neq 0)$ in $F^{n}$, and $S\left(F^{n}\right)$ the unit sphere in $F^{n}$ (i.e. $\left.S\left(F^{n}\right)=\left\{u \in F^{n}: u u^{*}=1\right\}\right)$. If $F=R$ and $n \geqq 3$ or $F=C$ or $H$ and $n \geqq 2$, then the image of the mapping $f: S\left(F^{n}\right) \rightarrow S\left(R^{2}\right)$ defined by

$$
f(u)=\left(\frac{u A u^{*}}{\left(\left(u A u^{*}\right)^{2}+\left(u B u^{*}\right)^{2}\right)^{1 / 2}}, \frac{u B u^{*}}{\left(\left(u A u^{*}\right)^{2}+\left(u B u^{*}\right)^{2}\right)^{1 / 2}}\right)
$$

is a closed circular arc of length $<\pi$.

THEOREM 2. Let $A$ and $B$ be two $n \times n$ hermitian matrices with elements in $F$ such that $\left(u A u^{*}\right)^{2}+\left(u B u^{*}\right)^{2}>0$ for all $u(\neq 0)$ in $F^{n}$. If $F=R$ and $n \geqq 3$ or $F=C$ or $H$ and $n \geqq 2$, then $A$ and $B$ can be diagonalized simultaneously (i.e. there exists a nonsingular $n \times n$ matrix $U$ with elements in $F$ such that $U A U^{*}$ and $U B U^{*}$ are diagonal matrices).

For the case $F=R$ and $n \geqq 3$, Theorem 2 has been proved by Greub [1, pp. 231-237], and Calabi [2] $]^{2}$ by different methods.

Received by the editors October 27, 1967.

1 The author wishes to thank Professor Y. C. Wong for suggesting the problem and for his advice and encouragement during the preparation of this paper.

2 The author is grateful to the referee for drawing his attention to this paper. 
2. A mapping from a sphere to the unit circle. We first prove a lemma.

Lemma 1. For any real numbers $a_{0}, \cdots, a_{4}, b_{0}, \cdots, b_{4}$ the following system of equations

$$
\begin{aligned}
a_{0}\left(x^{2}-y^{2}\right)+a_{1} x y+a_{2} x z+a_{3} y z+a_{4} z^{2} & =0, \\
b_{0}\left(x^{2}-y^{2}\right)+b_{1} x y+b_{2} x z+b_{3} y z+b_{4} z^{2} & =0,
\end{aligned}
$$

has nontrivial real solutions.

Proof. There are two cases.

Case 1. $a_{0} b_{1}-a_{1} b_{0}=0$. In this case we put $z=0$ in (1), and (1) becomes

$$
a_{0}\left(x^{2}-y^{2}\right)+a_{1} x y=0, \quad b_{0}\left(x^{2}-y^{2}\right)+b_{1} x y=0 .
$$

Since $a_{0} b_{1}-a_{1} b_{0}=0$, (2) has nontrivial real solutions. Hence (1) has nontrivial real solutions.

Case 2. $a_{0} b_{1}-a_{1} b_{0} \neq 0$. In this case we put $z=1$ in (1), and (1) becomes

$$
\begin{gathered}
a_{0}\left(x^{2}-y^{2}\right)+a_{1} x y+a_{2} x+a_{3} y+a_{4}=0, \\
b_{0}\left(x^{2}-y^{2}\right)+b_{1} x y+b_{2} x+b_{3} y+b_{4}=0 .
\end{gathered}
$$

Since $a_{0} b_{1}-a_{1} b_{0} \neq 0,(3)$ is equivalent to

(4) $x^{2}-y^{2}+a_{2}^{\prime} x+a_{3}^{\prime} y+a_{4}^{\prime}=0, \quad x y+b_{2}^{\prime} x+b_{3}^{\prime} y+b_{4}^{\prime}=0$,

where $a_{2}^{\prime}, a_{3}^{\prime}, a_{4}^{\prime}, b_{2}^{\prime}, b_{3}^{\prime}$ and $b_{4}^{\prime}$ are some real numbers. But (4) obviously has real solutions. Hence (1) has nontrivial real solutions.

We now prove Theorem 1 . Since $f$ is continuous and $S\left(F^{n}\right)$ is connected and compact, $f\left(S_{\left.\left(F^{n}\right)\right)}\right.$ is a closed circular arc. Therefore, Theorem 1 will be proved if we can show that, for any $(a, b)$ in $S\left(R^{2}\right)$, $(a, b)$ and $(-a,-b)$ cannot both belong to $f\left(S\left(F^{n}\right)\right)$.

Assume that there exists $\left(a_{0}, b_{0}\right)$ in $S\left(R^{2}\right)$ such that $\left(a_{0}, b_{0}\right)$ and $\left(-a_{0},-b_{0}\right)$ both belong to $f\left(S\left(F^{n}\right)\right)$. Then by the definition of $f$ there exist $u_{1}$ and $u_{2}$ in $F^{n} \backslash\{0\}$ such that $\left(a_{0}, b_{0}\right)=\left(u_{1} A u_{1}^{*}, u_{1} B u_{1}^{*}\right)$ and $\left(-a_{0},-b_{0}\right)=\left(u_{2} A u_{2}^{*}, u_{2} B u_{2}^{*}\right)$. Obviously, $u_{1}$ and $u_{2}$ are linearly independent over $F$. Since $F=R$ and $n \geqq 3$ or $F=C$ or $H$ and $n \geqq 2$, there exists $u_{3}$ in $F^{n}$ such that $u_{1}, u_{2}$ and $u_{3}$ are linearly independent over $R$. Now, for any $(x, y, z)$ in $R^{3}$ we have

$$
\begin{aligned}
\left(x u_{1}+y u_{2}\right. & \left.+z u_{3}\right) A\left(x u_{1}+y u_{2}+z u_{3}\right)^{*} \\
& =a_{0}\left(x^{2}-y^{2}\right)+a_{1} x y+a_{2} x z+a_{3} y z+a_{4} z^{2},
\end{aligned}
$$

and 


$$
\begin{aligned}
\left(x u_{1}+y u_{2}\right. & \left.+z u_{3}\right) B\left(x u_{1}+y u_{2}+z u_{3}\right)^{*} \\
& =b_{0}\left(x^{2}-y^{2}\right)+b_{1} x y+b_{2} x z+b_{3} y z+b_{4} z^{2},
\end{aligned}
$$

where the $a$ 's and $b$ 's are all real numbers; for example, $a_{1}=u_{1} A u_{2}^{*}$ $+u_{2} A u_{1}^{*}, b_{1}=u_{1} B u_{2}^{*}+u_{2} B u_{1}^{*}$. Therefore, by Lemma 1 , there exists $\left(x_{0}, y_{0}, z_{0}\right) \neq(0,0,0)$ in $R^{3}$ such that

$$
\left(x_{0} u_{1}+y_{0} u_{2}+z_{0} u_{3}\right) A\left(x_{0} u_{1}+y_{0} u_{2}+z_{0} u_{3}\right)^{*}=0,
$$

and

$$
\left(x_{0} u_{1}+y_{0} u_{2}+z_{0} u_{3}\right) B\left(x_{0} u_{1}+y_{0} u_{2}+z_{0} u_{3}\right)^{*}=0 .
$$

Since $u_{1}, u_{2}$ and $u_{3}$ are linearly independent over $R$, we obtain a contradiction to the hypothesis that $\left(u A u^{*}\right)^{2}+\left(u B u^{*}\right)^{2}>0$ for all $u(\neq 0)$ in $F^{n}$. Hence Theorem 1 is proved.

3. Simultaneous diagonalization of two hermitian matrices. Suppose that the conditions of Theorem 2 are satisfied. By Theorem $1, f\left(S\left(F^{n}\right)\right)$ is a closed circular arc of length $<\pi$. Let $(a, b)$ be the midpoint of this circular arc. Then if $u$ is any point in $S\left(F^{n}\right)$ and if $\theta(<\pi / 2)$ is the angle between the radii of $S\left(R^{2}\right)$ with end points $(a, b)$ and $f(u)$, we have

$$
a \frac{u A u^{*}}{\left(\left(u A u^{*}\right)^{2}+\left(u B u^{*}\right)^{2}\right)^{1 / 2}}+b \frac{u B u^{*}}{\left(\left(u A u^{*}\right)^{2}+\left(u B u^{*}\right)^{2}\right)^{1 / 2}}=\cos \theta>0 .
$$

Therefore, $a A+b B$ is positive definite, and Theorem 2 is proved by the following lemma:

Lemma 2. If $A$ and $B$ are two $n \times n$ hermitian matrices with elements in $F$ such that $a A+b B$ is positive definite for some $(a, b)$ in $R^{2}$, then $A$ and $B$ can be diagonalized simultaneously.

Proof. Since $a A+b B$ is a positive definite hermitian matrix, one of the $a$ and $b$, say $a$, is not zero and there exists a unitary matrix $U_{1}$ such that

$$
U_{1}(a A+b B) U_{1}^{*}=\operatorname{diag}\left(a_{1}, \cdots, a_{n}\right),
$$

where $a_{1}, \cdots, a_{n}$ are positive real numbers. (This is well known if $F=R$ or $C$; for example, see [3, pp. 12-13]. For $F=H$, it is proved in [4] and [5].) Let $U_{2}=\operatorname{diag}\left(1 / \sqrt{ } a_{1}, \cdots, 1 / \sqrt{ } a_{n}\right)$. Then

$$
U_{2} U_{1}(a A+b B) U_{1}^{*} U_{2}^{*}=I,
$$

where $I$ is the $n \times n$ identity matrix. Since $U_{2} U_{1} B U_{1}^{*} U_{2}^{*}$ is a hermitian matrix, there exists a unitary matrix $U_{3}$ such that 
(6) $U B U^{*}=$ diagonal matrix, where $U=U_{3} U_{2} U_{1}$.

From (5) and (6) it follows that

$$
U A U^{*}=1 / a\left(I-b U B U^{*}\right)=\text { diagonal matrix. }
$$

Thus Lemma 2 is proved.

ADDED IN PROOF. The author has just learned that, for the case $F=$ real closed field and $n \geqq 3$, Theorem 2 has been proved by Wonenburger [J. Math. Mech. 15 (1966), 617-622]; and for the case $F=R$ and $n \geqq 3$ or $F=C$ and $n \geqq 2$ by Kraljević [Glasnik Mat. Ser. III 1 (21) (1966), 57-63]. Their methods of proof are quite different from that of the author.

\section{REFERENCES}

1. W. H. Greub, Linear algebra, 2nd ed., Springer-Verlag, Berlin, 1963.

2. E. Calabi, Linear systems of real quadratic forms, Proc. Amer. Math. Soc. 15 (1964), 844-846. 1964.

3. C. Chevalley, Theory of Lie groups, Princeton Univ. Press, Princeton, N. J.,

4. J. Radon, Linear Scharen orthogonaler Matrizen, Abh. Math. Sem. Univ. Hamburg 1 (1922), 1-14.

5. H. C. Lee, Eigenvalues and canonical forms of matrices with quaternion coefficients, Proc. Roy. Irish Acad. Sect. A 52 (1949), 253-260.

UNIVERSITY OF HONG KONG 